\title{
La técnica de seguimiento ocular y el estudio de modelos tácitos mediante criterios subjetivos y conductuales
}

\section{The eye-tracking technique and the study of tacit models using subjective and behavioral criteria}

\section{A técnica de rastreamento ocular e o estudo de modelos tácitos usando critérios subjetivos e comportamentais}

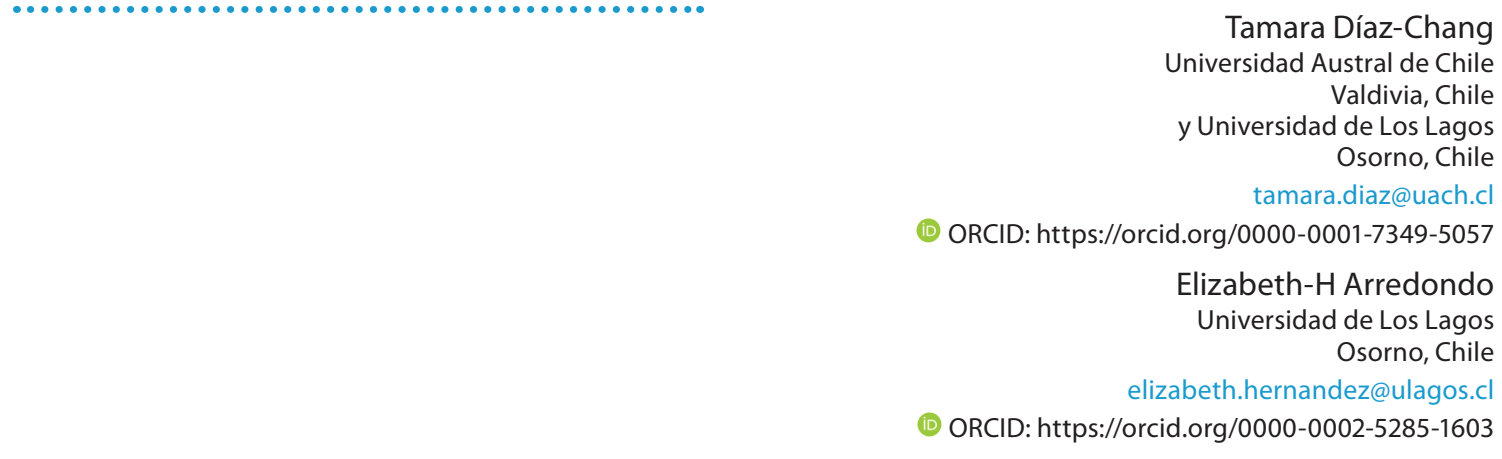

Recibido - Received - Recebido: 22 / 09 / 2021 Corregido - Revised - Revisado: 29 / 10 / 2021 Aceptado - Accepted - Aprovado: 12 / 11 / 2021

DOI: https://doi.org/10.22458/ie.v24i36.3894

URL: https://revistas.uned.ac.cr/index.php/innovaciones/article/view/3894

\begin{abstract}
Resumen: En este artículo se presentan los resultados de una investigación de enfoque mixto, donde se examina el movimiento ocular del estudiantado de pregrado de la Universidad Austral de Chile, mientras se resuelve un cuestionario donde aparecen modelos tácitos, relacionados con el infinito matemático, con el objetivo de determinar posibles correlaciones entre los parámetros de la actividad ocular y el nivel de dificultad de cada uno de estos modelos. Las categorías del nivel de dificultad se establecieron con base en dos tipos de criterios: uno subjetivo, mediante una evaluación realizada por los sujetos y uno conductual, relacionado con la obtención de la solución correcta. Se identificaron las correlaciones de estos criterios con los parámetros de actividad ocular, que se consideraron indicadores de esfuerzo mental. El análisis de los datos obtenidos permitió observar discrepancias en la categorización de los modelos tácitos, con base en criterios subjetivos y conductuales. Hubo una correlación negativa de los parámetros del movimiento ocular con las opiniones de los estudiantes, sobre el nivel de dificultad de las preguntas, mientras que se notó una fuerte correlación positiva y significativa entre la presencia de estos modelos y el nivel de dificultad, determinado por el porcentaje de respuestas correctas. A su vez, el porcentaje de respuestas correctas tuvo una fuerte correlación positiva y significativa con la mayoría de los parámetros de la actividad ocular. A partir de estos resultados, se concluye que, estos parámetros pueden tomarse como un índice del nivel de dificultad de los modelos tácitos presentes en una actividad.
\end{abstract}

Palabras clave: Modelos tácitos, seguimiento ocular, neuro-psicología, proceso cognitivo, aprendizaje, enseñanza de las matemáticas.

Abstract: This article presents the results of a mixed-approach research study, where the eye movement of the undergraduate students of the Austral University of Chile is examined while solving a questionnaire where tacit models appear, related to mathematical infinity, to determine possible correlations between the ocular activity parameters and the level of difficulty of each of these models. The categories of the level of difficulty were established based on two types of criteria: a subjective one, through an evaluation, carried out by the subjects, and a behavioral one, related to obtaining the correct solution. The correlations of these criteria with the ocular activity parameters were identified and considered indicators of mental effort. The analysis of the data obtained allowed us to observe discrepancies in the categorization of the tacit models based on subjective and behavioral criteria. There was a negative correlation of the eye movement parameters with students' opinions 
about question difficulty levels. In contrast, a strong positive and significant correlation was noted between the presence of these models and the level of difficulty, determined by the percentage of correct answers. In turn, the percentage of correct answers had a strong positive and significant correlation with most of the ocular activity parameters. These results conclude that these parameters can be used to index this activity's tacit model's difficulty level.

Keywords: Tacit models, eye tracking, neuropsychology, cognitive process, learning, mathematics teaching.

Resumo: Este artigo apresenta os resultados de uma pesquisa de abordagem mista, onde foi examinado o movimento ocular do aluno de graduação da Universidade Austral do Chile, enquanto se responde um questionário onde aparecem modelos tácitos, relacionados ao infinito matemático, visando determinar possíveis correlações entre os parâmetros de atividade ocular e o nível de dificuldade de cada um desses modelos. As categorias do grau de dificuldade foram determinadas a partir de duas categorias de critérios: uma subjetiva, através de uma avaliação realizada pelos sujeitos, e uma comportamental, relacionada à obtenção da solução correta. Foram identificadas as correlações desses critérios com os parâmetros de atividade ocular, considerados indicadores de esforço mental. A análise dos dados obtidos permitiu observar discrepâncias na categorização dos modelos tácitos, com base em critérios subjetivos e comportamentais. Houve correlação negativa dos parâmetros de movimento ocular com as opiniões dos alunos, sobre o nível de dificuldade das questões, enquanto uma forte correlação positiva e significativa foi observada entre a presença desses modelos e o nível de dificuldade, determinado pela porcentagem de respostas corretas. No que concerne, a porcentagem de respostas corretas houve uma forte correlação positiva e significativa com a maioria dos parâmetros da atividade ocular. Segundo os resultados, conclui-se que esses parâmetros podem ser tomados como um índice do nível de dificuldade dos modelos tácitos existentes em uma atividade.

Palavras-chave: modelos tácitos, rastreamento ocular, neuropsicologia, processo cognitivo, aprendizagem, ensino de matemática.

\section{INTRODUCCIÓN}

El infinito matemático, es uno de los conceptos más complejos a desarrollar por el estudiantado en cualquier nivel escolar; por ello, ha sido ampliamente estudiado desde diferentes perspectivas teóricas en educación matemática. A pesar de esto, no es posible afirmar que comprendemos los complicados procesos cognitivos que se desarrollan, en relación con su aprendizaje. Por ejemplo, Dubinsky, Weller, Mac Donald y Brown (2005) afirman que, dada su naturaleza, el sistema cognitivo de un individuo no puede apropiarse del concepto infinito a través de manipulaciones operativas, representadas por proposiciones formalmente rigurosas. Existen numerosos trabajos (e.g. Arrigo y D'Amore, 2004; Fischbein, 2001) que dan cuenta de la dificultad y complejidad que presenta, en este caso, el obstáculo epistemológico (Bachelard, 1938/2004).

En particular, los resultados de varios estudios (e.g. Dubinsky, Weller, McDonald y Brown, 2005; Fischbein, 2001; Mena-Lorca, Montoya-Delgadillo, Morales y Parraguez, 2015) muestran que muchas de las paradojas sobre el infinito (Bolzano, 1991), surgen como convicciones intuitivas que provocan dificultades en el proceso de emergencia de los significados de este concepto. Por ejemplo, una convicción intuitiva muy común entre el estudiantado, es pensar que en un segmento más largo, hay más puntos que en un segmento más corto (e.g. D'Amore y Martini, 1997; Tall, 1981), lo que D'Amore (2011) denomina dependencia de los cardinales transfinitos a hechos relativos a la medida.

Fishbein (2001) argumenta que este tipo de convicciones intuitivas como la dependencia, constituyen modelos tácitos, implícitos e inconscientes, que aparecen cuando tratamos con conceptos que son demasiado abstractos o complejos. En estas circunstancias, tenemos una tendencia natural a pensar en términos de modelos mentales simplificados, que nos ayudan a representar a las identidades originales, con el objetivo de facilitar y estimular la tarea de comprensión o resolución y luego, se vuelven implícitos o tácitos, puesto que perdemos conciencia de ellos, controlando nuestro razonamiento de manera inconsciente para nosotros e impidiendo la comprensión adecuada de estos conceptos. 
En particular, en este trabajo nos centraremos en el estudio de seis de estos modelos tácitos, que ya han sido caracterizados, ampliamente, en la literatura y que son adoptados con frecuencia, cuando se estudian temas relacionados con el infinito matemático, en el nivel universitario: la inagotabilidad, la indefinición, la divergencia, la dependencia, lo inalcanzable y el modelo acotado-finito. Para una revisión detallada y exhaustiva, ver por ejemplo, Belmonte y Sierra (2011). Claramente, el estudio de estos modelos y esquemas inconscientes, es de especial importancia para la comprensión de los obstáculos y dificultades que surgen en este caso. Marshall (2002) señala que la dificultad de un problema, se expresa en términos del esfuerzo cognitivo necesario para resolverlo. Es decir, los procesos o recursos cognitivos que se activan en la resolución de un problema, que pueden ser índices de su nivel de su dificultad, están conectados con el concepto de carga cognitiva. Young y Stanton (2001) argumentan que la carga cognitiva de una tarea, está dada por el nivel de recursos de atención que se necesita para cumplir con criterios de desempeño objetivos y subjetivos.

Tanto factores internos como externos influyen en el nivel de carga cognitiva en cada caso. Los primeros, están vinculados con el esfuerzo mental que requiere el procesamiento de la información, teniendo en cuenta su estructura, complejidad y especificidad, mientras que los segundos, están vinculados a la forma en que se presenta la información (Sweller, 1994).

Por lo general, las medidas utilizadas para medir el esfuerzo mental se dividen en tres grupos principales: subjetivo, relativo a una evaluación individual del grado de complejidad de la actividad; conductual, relacionado con la calidad del desempeño en la actividad; y neuro-fisiológicos, entre los que se encuentran la medición de la frecuencia cardíaca, la respiración, las técnicas de neuroimagen y los métodos de seguimiento ocular (May et al., 1990).

En particular, en este trabajo se utilizan los parámetros del movimiento ocular, entre las medidas neurofisiológicas, como indicadores del esfuerzo mental. Notemos que los métodos de seguimiento ocular asumen que el aprendizaje está relacionado, principalmente, con la percepción y el procesamiento de los estímulos del entorno. El sentido más importante que proporciona la mayor cantidad de información, es el sentido de la vista y por esto, el estudio de la actividad visual mediante la técnica del seguimiento ocular (eye-tracking), se ha convertido en un tema de interés durante los últimos años, para las investigaciones en educación matemática, que incorporan los avances de la neurociencia cognitiva (Strohmaier, MacKay, Obersteiner y Reiss, 2020).

Las actividades de la percepción visual son responsables del análisis, síntesis e interpretación de imágenes, transmitidas desde la retina por los nervios ópticos, al centro visual de la corteza cerebral. El sistema de atención decide seleccionar un objeto y dirigir la mirada hacia él, en promedio de tres veces por segundo. Como resultado, se considera que la técnica del seguimiento ocular proporciona un reflejo preciso de las interacciones, entre los procesos cognitivos y los estímulos visuales externos (Soluch y Tarnowski, 2013).

La mayoría de los rastreadores oculares, utilizan diodos emisores de luz infrarroja para iluminar el ojo y utilizan la relación, entre el reflejo de la córnea y el centro de la pupila, para identificar la dirección de la mirada. Generalmente, se pueden dividir en dos tipos: remotos y portátiles. Los laboratorios utilizan sistemas remotos y una unidad de interfaz gráfica integrada, que proporciona información en tiempo real, en una pantalla. Las especificaciones técnicas de los dispositivos son diversas. La frecuencia de muestreo tradicional es de $60 \mathrm{~Hz}$, pero existen muchos dispositivos que usan una frecuencia mayor. Notemos que la alta frecuencia de medición no garantiza su precisión. Se requiere un procedimiento de calibración del dispositivo para calcular el mapeo, entre las mediciones y la orientación del ojo (Ramanauskas, 2006). Durante la calibración, el participante se fija en 7-13 puntos en la pantalla, mientras el rastreador ocular monitorea el ojo. 
Las medidas de seguimiento ocular más utilizadas son: la dilatación de la pupila, el conteo de fijaciones y la duración de la fijación. La fijación es un estado, en el que la mirada se fija en el objeto observado. La duración de la fijación depende, únicamente, del procesamiento de la información y es aproximadamente, de 150 milisegundos a 1500 milisegundos (Steinman, 2004). Entre las fijaciones, se considera el movimiento sacádico, es decir, un movimiento rápido, escalonado, en salto, del globo ocular, conectado con cambios en los puntos de foco ocular.

En las últimas investigaciones realizadas, se puede notar un creciente interés en los cambios de dilatación de la pupila. Algunos consideran que estos cambios, son importantes en el análisis de experimentos e índices de procesamiento de información (Poole y Ball, 2006). En condiciones de iluminación fija, la pupila aumenta de diámetro, cuando aumenta la dificultad de la tarea realizada. Sin embargo, la susceptibilidad de esta medida a factores externos, como las condiciones de iluminación, constituye una limitación experimental.

A partir de los antecedentes anteriores, los objetivos específicos de esta investigación consisten, en determinar las posibles correlaciones entre los parámetros de la actividad ocular y, la evaluación del nivel de dificultad de las preguntas; entre los parámetros de la actividad ocular y el porcentaje de respuestas correctas; $y$ entre la presencia de modelos tácitos, la evaluación del nivel de dificultad y el porcentaje de respuestas correctas; que nos permitan a su vez, lograr el objetivo general de investigación: determinar posibles correlaciones entre todos estos parámetros, y el nivel de dificultad de los modelos tácitos que se presentan en el estudio del infinito matemático en cada caso.

\section{MATERIALES Y MÉTODOS}

\section{Enfoque de investigación}

Se usó un enfoque de investigación mixto. Se realizó un análisis cuantitativo de parámetros de la actividad ocular, combinado con el análisis cualitativo del nivel de dificultad de las preguntas, establecido por medio de criterios subjetivos y conductuales, y la aparición de modelos tácitos.

\section{Participantes}

Participaron en el experimento 59 estudiantes de pregrado de 19 a 25 años de edad, de la Facultad de Ciencias de la Universidad Austral de Chile, entre ellos 22 estudiantes femeninas y 37 masculinos, 9 eran estudiantes de la licenciatura en Matemática y los demás, pertenecían a distintas carreras de la Facultad de Ciencias. La elección de la muestra fue no probabilista, por conveniencia. Los datos de medición de 7 sujetos (estudiantes de Ciencias), fueron rechazados por razones técnicas y 52 casos, calificaron para un análisis más detallado. Dado que en esta investigación se busca una correlación, entre la evaluación subjetiva y los parámetros del movimiento ocular, la vista de los sujetos era normal o estaba corregida a normal. Todos dieron su consentimiento por escrito para participar en el experimento $y$, se tomaron todas las medidas establecidas por el protocolo de la Universidad Austral de Chile, ante la emergencia sanitaria. 


\section{Procedimiento}

Para realizar el experimento, el estudiantado asistió a un laboratorio del Centro Interdisciplinario de Estudios del Sistema Nervioso, CISNe, de la Universidad Austral, mientras la profesora se encontraba conectada en línea. Considerando que factores, como el estrés, la presión del tiempo o el ruido, y otros provenientes del entorno pueden influir en la carga cognitiva de un individuo, se intentó eliminarlos o minimizarlos. Por esta misma razón y debido a que, durante el experimento no se proporcionó un control estricto de las condiciones de iluminación, los parámetros relacionados con la dilatación de la pupila, no fueron medidos ni considerados. Antes y después de las mediciones de seguimiento ocular, el estudiantado mantuvo conversaciones virtuales a través de Zoom con la profesora, durante las cuales se les informó sobre el experimento. El objetivo de estas conversaciones fue reducir el nivel de estrés, inducir una actitud positiva en los estudiantes y motivarlos a involucrarse en el proceso de resolución. Después de la conversación introductoria, los estudiantes completaron los cuestionarios, mientras la profesora permanecía en línea, dialogando con los estudiantes.

El cuestionario utilizado se muestra en la Figura 1 y se diseñó teniendo en cuenta, estos seis modelos tácitos presentes en el estudio, del infinito matemático según Belmonte y Sierra (2011), y basado en la paradoja de Aquiles y la tortuga de Zenón de Elea en el siglo V a.C. (Bolzano, 1991). Este cuestionario fue analizado en un estudio previo, mostrándose los modelos tácitos adoptados por los estudiantes, en cada una de las preguntas (Díaz-Chang y Arredondo, 2021a). Cada pregunta requirió un análisis del contenido, se evaluó su confiabilidad, consistencia interna y potencial de discriminación. Además, el cuestionario fue validado mediante el pilotaje y la triangulación de contenidos y de expertos, antes de quedar conformado en su versión final para ser utilizado en el experimento. Para conocer detalles de este análisis, ver Díaz-Chang y Arredondo (2021b). Dicho cuestionario fue presentado a los estudiantes, con el siguiente enunciado:

Aquiles, el héroe griego de la guerra de Troya decide competir en una carrera con la lenta tortuga, a lo largo de una pista recta de longitud distinta de cero, que va desde un punto $A$ hasta un punto $B$. Como Aquiles le dobla a la tortuga en velocidad, este en un gesto de generosidad le permite a la tortuga iniciar la carrera en la mitad del trayecto. Pero cuando Aquiles ha llegado a la mitad del trayecto, la tortuga ya se ha desplazado a la mitad del trayecto que resta. Así, en la siguiente etapa, cuando Aquiles ha llegado al punto donde se encontraba la tortuga, ella ya se ha desplazado nuevamente a la mitad del camino que restaba. Y así sucesivamente, no importa cuántas etapas sucedan, la tortuga siempre le llevará una ventaja a Aquiles, equivalente a la mitad del camino que quedaba en la etapa anterior. Suponiendo que ambos corren a velocidad constante, el problema consiste en determinar quién ganó la carrera. 
Figura 1. Cuestionario "Aquiles y la tortuga"

\section{Cuestionario Aquiles y la tortuga}

1. Cuando Aquiles llegue al punto $C$, ya la tortuga se ha desplazado hasta el punto $D$, pero cuando Aquiles llegue al punto $D$, ya la tortuga se ha desplazado hasta el punto $E$. A su vez, cuando Aquiles llegue al punto $E$, ya la tortuga se ha desplazado hasta el punto $F$, y cuando Aquiles llegue al punto $F$, ya la tortuga se ha desplazado hasta el punto $G$.

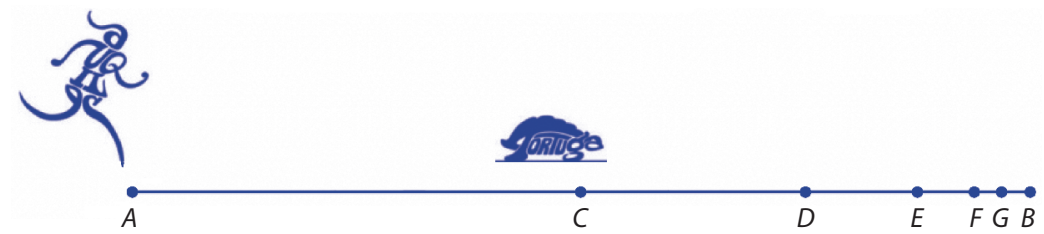

En la siguiente tabla aparece la distancia total recorrida por Aquiles desde la etapa 1 hasta la 5.

Etapa $\frac{\bar{A} \text { Distancia total recorrida por Aquiles }}{1}$
$2 \frac{\frac{\bar{A}}{2}}{2}+\frac{\overline{A B}}{4}$
$3 \frac{\frac{\overline{A B}}{2}+\frac{\overline{A B}}{4}+\frac{\overline{A B}}{8}}{4} \frac{\frac{\overline{A B}}{2}+\frac{\overline{A B}}{4}+\frac{\overline{A B}}{8}+\frac{\overline{A B}}{16}}{5} \frac{\frac{\bar{A}}{2}}{5}+\frac{\overline{A B}}{4}+\frac{\overline{A B}}{8}+\frac{\overline{A B}}{16}+\frac{\overline{A B}}{32}$

Continuando este proceso iterativo, ¿cuál será la distancia total recorrida por Aquiles en la $n$-ésima etapa?

2. ¿Cuál será la distancia total recorrida por la tortuga en la $n$-ésima etapa?

3. ¿Cuál será la distancia que los separa en la n-ésima etapa?

4. ¿Alcanzará en algún momento Aquiles a la tortuga?

5. ¿Será posible efectuar la suma infinita $\frac{1}{2}+\frac{1}{4}+\frac{1}{8}+\frac{1}{16}+\frac{1}{32}+\ldots \frac{1}{2^{n}}+\ldots$ y afirmar que su resultado es igual a 1?

6. ¿Será posible verificar que la suma $\frac{1}{4}+\frac{1}{8}+\frac{1}{16}+\frac{1}{32}+\ldots+\frac{1}{2^{n}}+\ldots=\frac{1}{2}$ ?

7. Teniendo en cuenta sus respuestas a las preguntas anteriores, ¿sucederá que $1 / 2^{n}=0$ en algún momento?

8. Finalmente, ¿quién ganará la carrera?

Fuente: Elaboración propia.

Según el análisis mencionado y realizado anteriormente, a continuación se muestran (ver Tabla 1) los modelos tácitos en relación con el infinito matemático que se presentaron en el razonamiento de los sujetos, a partir de cada una de las preguntas planteadas.

TABLA 1

Modelos tácitos presentes en el razonamiento (por cada pregunta)

\begin{tabular}{cl} 
Pregunta & \multicolumn{1}{c}{ Modelos tácitos } \\
$\mathrm{N} .{ }^{\circ} 1$ & - \\
$\mathrm{N} .{ }^{\circ} 2$ & - \\
$\mathrm{N} .{ }^{\circ} 3$ & - \\
$\mathrm{N} .{ }^{\circ} 4$ & inagotabilidad, dependencia, divergencia, indefinición \\
$\mathrm{N} .{ }^{\circ} 5$ & inagotabilidad, divergencia, indefinición, inalcanzable \\
$\mathrm{N} .{ }^{\circ} 6$ & inagotabilidad, divergencia, indefinición, inalcanzable \\
$\mathrm{N} .{ }^{\circ} 7$ & inagotabilidad, divergencia, indefinición, inalcanzable \\
$\mathrm{N} .{ }^{\circ} 8$ & inagotabilidad, dependencia, divergencia, indefinición, acotado-finito \\
\hline
\end{tabular}

Fuente: Elaboración propia. 
Cada estudiante, de manera independiente, realizó el experimento de seguimiento ocular en el laboratorio, después que se le proporcionara la instrucción introductoria. Cada uno de ellos llevó a cabo el proceso de calibración y las actividades de prueba necesarias, para garantizar el desempeño apropiado de las mediciones. El tiempo para la resolución del cuestionario, no fue limitado y cada estudiante trabajó a su propio ritmo, seleccionando la respuesta correcta de una lista de categorías disponibles usando el mouse, y de manera adicional, se les pidió que comentaran oralmente las justificaciones a sus respuestas, en diálogo con la profesora.

\section{DISPOSITIVO DE SEGUIMIENTO OCULAR Y PARÁMETROS OCULARES:}

En el experimento se utilizó un dispositivo de seguimiento ocular Tobii X2-60, proporcionado por Tobii Pro, que registra un flujo de datos con resolución de tiempo de $60 \mathrm{~Hz}$, registrando las coordenadas de la posición ocular (una medida relativa y otra absoluta), y los parámetros de movimientos sacádicos y fijaciones. La precisión espacial del dispositivo es de $0,3^{\circ}$, el retardo de cálculo menor a 0,5 milisegundos y el retardo del sistema inferior, a 2 milisegundos.

A continuación, se muestra, detalladamente, la descripción de los parámetros medidos, que son mostrados mediante los mapas de escaneo visual y los mapas de color:

- Duración media, total, frecuencia y conteo total de fijaciones: Según los resultados obtenidos en numerosas investigaciones (ver por ejemplo, Francuz, 2013), una mayor duración de la fijación en un área de la interface, está relacionada con la dificultad para interpretar la información presente o una mayor implicación en su exploración. Por el contrario, los elementos de la interface que son comprensibles y los que no aportan información semántica significativa, se caracterizan por una duración de fijación más corta (Henderson y Hollingworth, 1998). Esta regularidad, también ha sido confirmada por numerosas investigaciones de seguimiento ocular en las que los participantes resolvieron problemas de matemáticas. Sobre esta base, se ha concluido que cuanto más complejo es el problema, mayor es la duración de la fijación y mayor es su conteo (Rayner, 1998). Por otro lado, se ha notado que la duración promedio de la fijación es mayor cuando se ven imágenes de carácter abstracto, en comparación con imágenes figurativas, lo que indica que las imágenes abstractas están sujetas a un procesamiento visual más profundo y pueden ser una fuente de una mayor carga cognitiva, relacionada con más difícil percepción e interpretación (Bałaj y Szubielska, 2014).

- Frecuencia y conteo total de movimientos sacádicos: Normalmente, los períodos de fijación se ven interrumpidos por los movimientos sacádicos. Estos movimientos se detectan, por desviaciones en la posición ocular por encima de $0,1^{\circ}$, de velocidad mínima de $30^{\circ}$ por segundo y aceleración mínima de $8000^{\circ}$ por segundo al cuadrado, que se sostienen por lo menos, durante 4 milisegundos. Las relaciones entre los parámetros de fijación y los índices de carga cognitiva no son inequívocas, debido a estos movimientos sacádicos y pueden depender del tipo de tarea y las condiciones en las que se realiza una tarea (Backs y Walrath, 1992). Para corregir esto, los investigadores Goldberg y Kotval (1999) utilizan la razón entre la duración de la fijación y la cantidad de movimientos sacádicos como índice, que proporciona información sobre la prevalencia de procesos de procesamiento de información, sobre procesos de búsqueda visual. Dada una misma cantidad de movimientos sacádicos, una razón mayor indica que hubo mayor procesamiento de la información que en el caso en que se tiene una razón menor.

- Longitud y trayectoria de escaneo visual, sobre las áreas de interés de la interface: En este caso, se quiere estudiar la manera en que la información, es explorada visualmente por los estudiantes y el modo en que la representación como un todo, se les revela mediante este escaneo de exploración. Las áreas de interés permiten identificar áreas de la interface, donde se presenta información 
relevante y los registros, mediante los que esta información se presenta (Rayner, 1998). Estos parámetros serán medidos para examinar el comportamiento general de escaneo, puesto que no estaremos interesados en ninguna área de interés en específico, ni en los detalles de las transiciones entre ellas y solo, observaremos la longitud total de las trayectorias sobre la interface.

Por último, los resultados de las mediciones de estos parámetros, se procesaron utilizando el software Tobii Pro Lab, que nos entrega el procesamiento estadístico de las mediciones dadas por los mapas de calor y de las trayectorias visuales de los sujetos.

\section{UNA EVALUACIÓN DEL NIVEL DE DIFICULTAD DE LAS PREGUNTAS POR PARTE DE LOS SUJETOS}

Al final del experimento, los sujetos evaluaron el nivel de dificultad de las preguntas, respondiendo a una encuesta con el siguiente enunciado: Evaluar en la escala de 0 a 10 (donde 0 - muy fácil, 10 - muy difícil), en qué medida las preguntas que resolvió fueron fáciles - difíciles. Para evitar errores, en cada una de las preguntas, se presentó una imagen de la diapositiva original del cuestionario. El nivel de dificultad se midió, utilizando la escala de 11 puntos de Likert y sobre esta base, se determinaron las siguientes categorías (ver Tabla 2).

TABLA 2

Categorías asumidas para el nivel de dificultad de las preguntas

\begin{tabular}{cc} 
Rango de puntos & Nivel de dificultad de la pregunta \\
$0-2$ & muy fácil (1) \\
$2-4$ & fácil (2) \\
$4-6$ & moderadamente difícil (3) \\
$6-8$ & difícil (4) \\
$8-10$ & muy difícil (5) \\
\hline
\end{tabular}

\section{DISCUSIÓN DE RESULTADOS}

Para analizar los datos de la medición, se utilizó un análisis de varianza con una medición repetida, donde el tipo de pregunta que se presenta es el factor (intragrupo) de la medición repetida y la carrera del estudiante evaluado, es el factor intergrupal. Las variables dependientes fueron: la evaluación del nivel de dificultad de las preguntas, el porcentaje de soluciones correctas de las soluciones dadas y los parámetros del movimiento ocular seleccionados.

\section{EVALUACIÓN DEL NIVEL DE DIFICULTAD DE LAS PREGUNTAS}

Sobre la base de los valores de estadísticas descriptivas básicas (ver Tabla 3), se puede afirmar que entre las preguntas presentadas, ninguna fue categorizada en las dos categorías extremas: muy fácil o muy difícil. Se categorizó como difícil la pregunta n. 7 (media $=7,71$ ), moderadamente difícil las preguntas n. 4 (media $=4,35)$ y n. 8 (media $=5,12) ;$ y como fáciles, las preguntas n. ${ }^{\circ} 1$ (media $\left.=2,64\right), n .{ }^{\circ} 2$ (media $=$ $3,51), n .{ }^{\circ} 3$ (media $\left.=3,58\right), n .^{\circ} 5$ (media $\left.=3,71\right)$ y la pregunta n. 6 (media $\left.=3,75\right)$. 
TABLA 3

Evaluación del nivel de dificultad de la pregunta, por parte de los estudiantes $(\mathrm{N}=52)$

\begin{tabular}{|c|c|c|c|}
\hline Pregunta & Media & D.E. & Nivel de dificultad \\
\hline $\mathrm{N}^{0} 1$ & 2,64 & 2,33 & fácil (2) \\
\hline N. ${ }^{\circ} 2$ & 3,51 & 2,53 & fácil (2) \\
\hline N. 3 & 3,58 & 2,88 & fácil (2) \\
\hline N. ${ }^{\circ} 4$ & 4,35 & 2,71 & moderadamente difícil (3) \\
\hline N. .5 & 3,71 & 2,63 & fácil (2) \\
\hline N. 6 & 3,75 & 2,59 & fácil (2) \\
\hline N..$^{\circ}$ & 7,01 & 2,89 & difícil (4) \\
\hline N. ${ }^{\circ} 8$ & 5,12 & 2,75 & moderadamente difícil (3) \\
\hline
\end{tabular}

El análisis de varianza mostró, que las diferencias en la evaluación del nivel de dificultad, son significativas $[F(7,364)=1.65, p<0,05]$ y la prueba de Scheffé $(p<0,01)$ confirmó la diferencia significativa entre la pregunta $n .{ }^{\circ} 7$ y el resto, excepto la pregunta $n .{ }^{\circ} 8$ que fue significativamente, más difícil que la pregunta n. ${ }^{\circ} 1(p<0,01)$.

Los resultados se presentan en la Figura 2, donde además se muestran las diferencias entre los dos grupos, que también resultaron ser significativas $[F(1,52)=2,37, p<0,01]$. El estudiantado de carreras en Ciencias (media $=4,61$ ), consideró las tareas mucho más difíciles que el estudiantado de Matemáticas (media $=2,29$ ).

Figura 2. Evaluación del nivel de dificultad de la pregunta - diferencias entre los grupos: Matemáticas y Ciencias

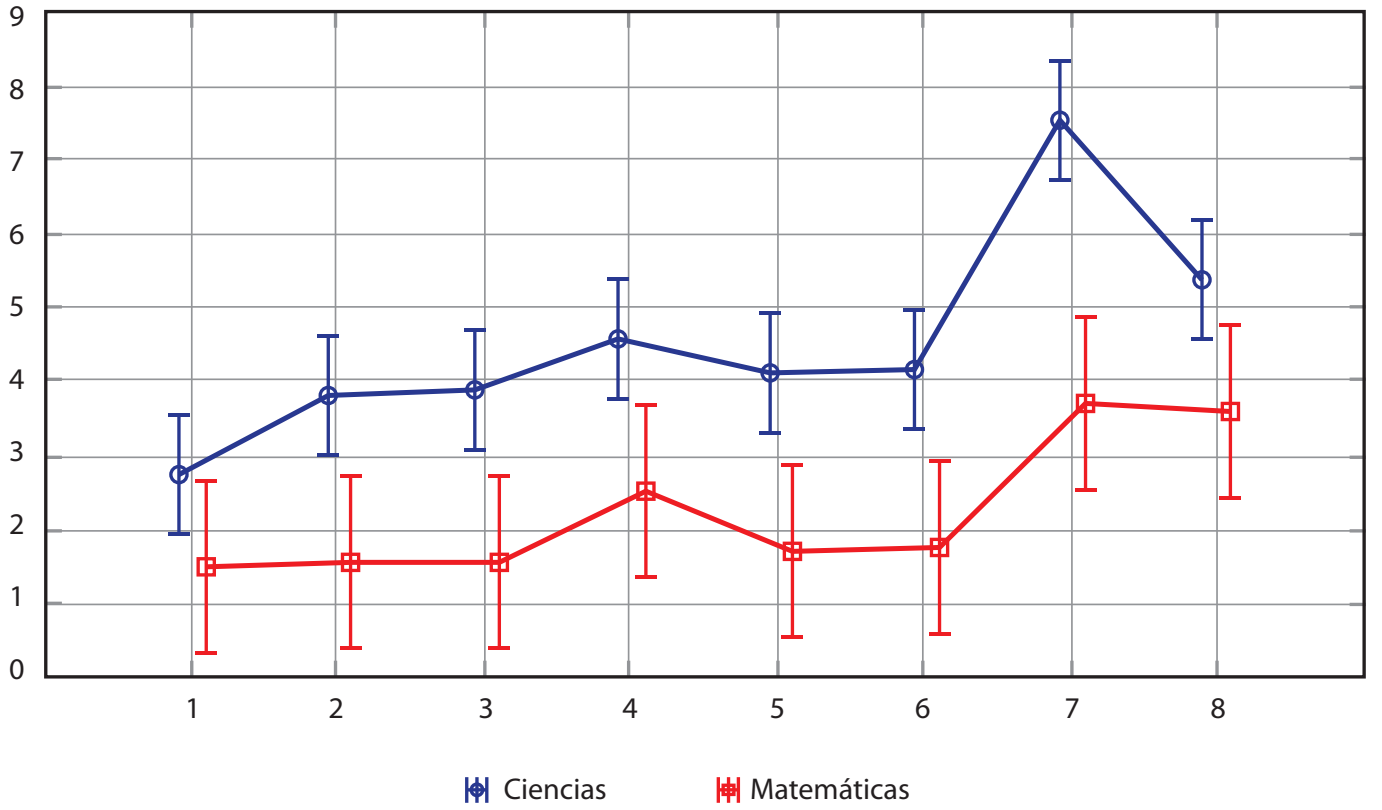

Fuente: Elaboración propia. 


\section{PORCENTAJE DE RESPUESTAS CORRECTAS COMO INDICADOR DEL NIVEL DE DIFICULTAD DE LAS PREGUNTAS}

El segundo criterio que se consideró para determinar el nivel de dificultad de las preguntas, fue el porcentaje de respuestas correctas obtenidas (criterio conductual). En la Tabla 4 se muestra la escala de dificultad utilizada.

TABLA 4

Categorías asumidas del nivel de dificultad de las preguntas

\begin{tabular}{cc} 
\% respuestas correctas & Nivel de dificultad de la pregunta \\
$0-19$ & muy difícil (5) \\
$20-39$ & difícil (4) \\
$40-59$ & moderadamente difícil (3) \\
$60-79$ & fácil (2) \\
$80-100$ & muy fácil (1) \\
\hline
\end{tabular}

Como se muestra en la Tabla 5, el porcentaje más bajo de respuestas correctas, se obtuvo para la pregunta n. $^{\circ}(15 \%)$, mientras que las preguntas n. 4 y n..$^{\circ}$ le siguen en orden (ambas con $\left.29 \%\right)$. En el grupo de preguntas difíciles, también estaba la n. 6 (31\%). El mayor porcentaje de respuestas correctas se encontró para la pregunta.$^{\circ} 1(65 \%)$ y la pregunta ${ }^{\circ}{ }^{\circ} 2(60 \%)$.

TABLA 5

Porcentaje de respuestas correctas como criterio del nivel de dificultad de la pregunta $(\mathrm{N}=52)$

\begin{tabular}{|c|c|c|}
\hline Pregunta & $\begin{array}{c}\% \text { respuestas } \\
\text { correctas }\end{array}$ & Nivel de dificultad de la pregunta \\
\hline N. ${ }^{\circ} 8$ & 15 & muy difícil (5) \\
\hline N. ${ }^{\circ} 7$ & 29 & \multirow{3}{*}{ difícil (4) } \\
\hline N. ${ }^{\circ} 4$ & 29 & \\
\hline N. ${ }^{\circ} 6$ & 31 & \\
\hline N.. 5 & 46 & \multirow{2}{*}{ moderadamente difícil (3) } \\
\hline $\mathrm{N}^{\circ} .3$ & 50 & \\
\hline N.. 2 & 60 & \multirow{2}{*}{ fácil (2) } \\
\hline N. ${ }^{0} 1$ & 65 & \\
\hline
\end{tabular}

El análisis de varianza mostró que había diferencias significativas, en el número de respuestas correctas $[F(7,364)=9,31, p<0,001]$, y la prueba de Scheffé confirmó diferencias significativas, en los resultados obtenidos por los sujetos entre la pregunta $n .^{\circ} 1$ y todas las demás $(p<0,01)$, excepto la pregunta $n .^{\circ} 2$ que fue significativamente más fácil que la $n^{\circ} 8(\mathrm{p}<0,01)$. 
Figura 3. Porcentaje de respuestas correctas - diferencias entre los grupos: Matemáticas y Ciencias

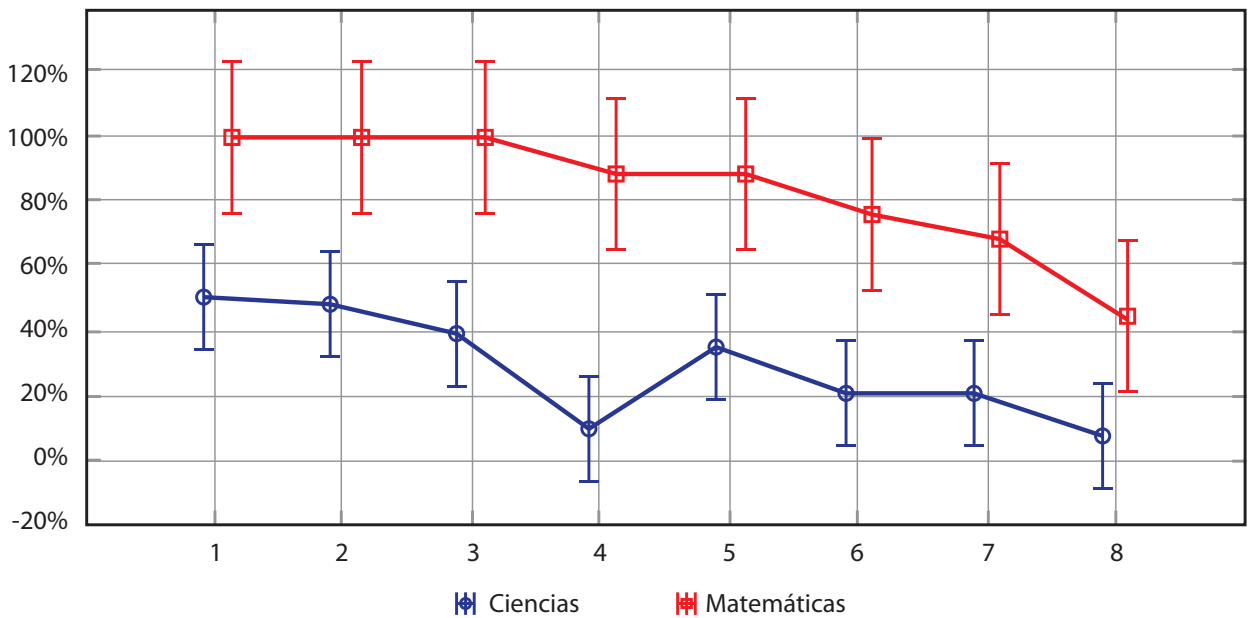

Fuente: Elaboración propia.

El porcentaje de respuestas correctas en el grupo de las carreras de Ciencias (media $=0,32$ ), fue más de dos veces menor que el del grupo de Matemáticas (media $=0,83$ ). Esta diferencia fue significativa $[F(1,52)=2,72, p<0,01]$. Las diferencias entre los grupos se presentan en la Figura 3. Como puede verse, las mayores discrepancias, se observan en las preguntas n. 3 (media grupo de Ciencias $=0,4$, media grupo de Matemáticas $=1,00$ ) y n. 4 (media grupo de Ciencias $=0,16$, media grupo de Matemáticas $=0,89$ ).

Los resultados obtenidos con base en este indicador resultaron ser, en general, consistentes con los obtenidos, según el criterio subjetivo de evaluación discutido anteriormente (ver comparación en la Figura 4). Las mayores diferencias se dieron para las preguntas n. 6 y n. 8 . La pregunta n. 6 fue "fácil", aunque obtuvo el segundo indicador más bajo de respuestas correctas. Una contradicción similar ocurrió en el caso de la pregunta . $^{\circ} 8$, que obtuvo el menor porcentaje de respuestas correctas de todo el cuestionario y fue la única pregunta categorizada como "muy difícil", según este criterio, pero según la opinión de los sujetos, es una pregunta solo "moderadamente difícil". Notemos que las divergencias obtenidas son compatibles con los resultados de otros estudios, en los que se han utilizado medidas subjetivas y conductuales (ver por ejemplo, May y colaboradores (1990).

Figura 4. Nivel de dificultad de la pregunta según los dos criterios determinados, en comparación con la cantidad de modelos tácitos presentes

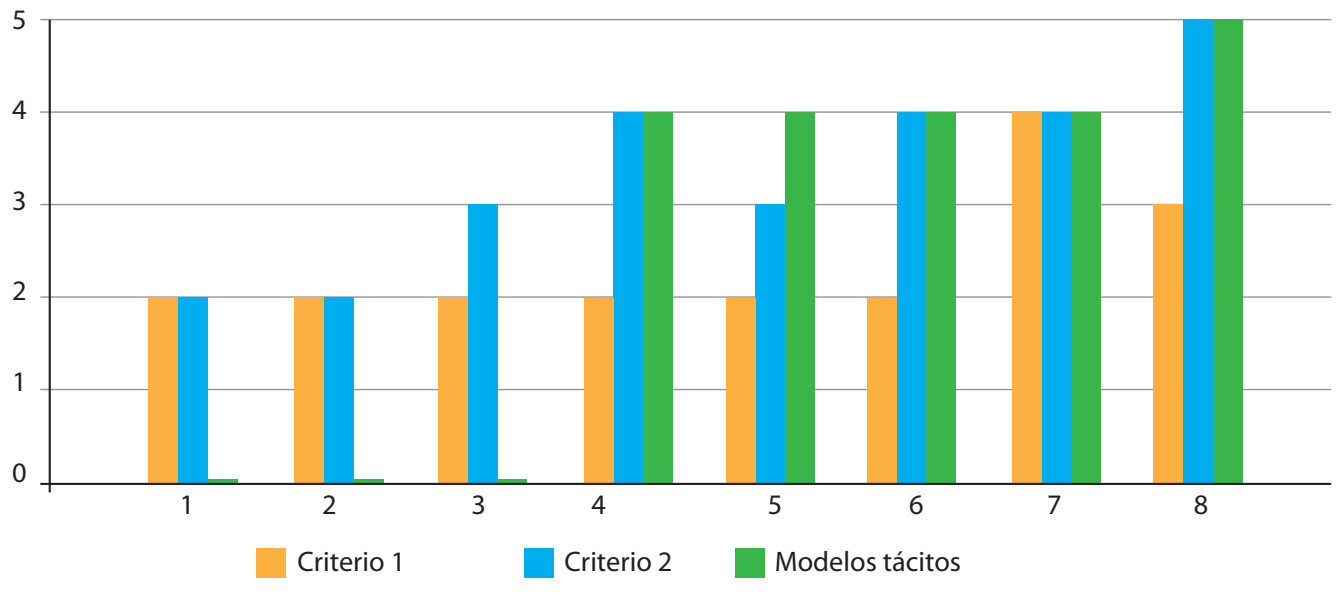

Fuente: Elaboración propia. 
La pregunta n.ㄱ, evaluada como la más "difícil" según el criterio subjetivo, presenta cuatro modelos tácitos (inagotabilidad, divergencia, indefinición, inalcanzable). En las preguntas evaluadas como "moderadamente difíciles" ( . $^{\circ} 4$ y n. ${ }^{\circ}$ ) también aparecen tres de estos modelos (inagotabilidad, divergencia, indefinición). Notemos además, que las preguntas "fáciles" (n.⒈, n. 2 2, n. ${ }^{\circ}$ ) no implican la aparición de ningún modelo. Por otra parte, la distribución de los resultados obtenidos, según el porcentaje de respuestas correctas refleja el nivel de dificultad de las preguntas, en concordancia con la cantidad de modelos tácitos presentes.

Excepto en el caso de las preguntas $\mathrm{n} .^{\circ} 1, \mathrm{n}^{\circ} 2 \mathrm{y} \mathrm{n} .^{\circ} 3$ (donde no hay presencia de modelos tácitos) y en el caso de la pregunta . $^{\circ}$, se percibe el nivel de dificultad de las preguntas, por debajo del nivel de dificultad indicado por la medición objetiva del porcentaje de respuestas correctas. Este hecho constituye una evidencia de la aparición inconsciente, de estos modelos erróneos de razonamiento.

La correlación entre el valor medio del nivel de dificultad dado, para todo el conjunto de preguntas y el porcentaje de respuestas correctas es fuerte $(r=-0,71)$ y significativa $(p<0,025)$. El hecho de que el índice de correlación sea negativo, significa que cuanto más alto se evalúa el nivel de dificultad de la pregunta, menor es el porcentaje de respuestas correctas que se obtiene.

Por otro lado, la correlación entre el valor medio del nivel de dificultad dado y la cantidad de modelos tácitos presente en cada pregunta, también es fuerte $(r=0,61)$ y significativa $(p<0,025)$. El porcentaje de respuestas correctas proporcionadas y la cantidad de estos modelos presente en cada pregunta, están fuertemente correlacionados $(r=-0,89)$ y es significativa $(p<0,025)$. Como ya hemos mencionado, el hecho de que el índice de correlación sea negativo, significa que cuanto mayor sea el número de modelos tácitos que aparecen asociados a una pregunta, menor es el porcentaje de respuestas correctas que se obtiene.

\section{LOS PARÁMETROS DE LA ACTIVIDAD OCULAR COMO INDICADORES DEL ESFUERZO MENTAL:}

Los parámetros de la actividad ocular que se consideran como indicadores del procesamiento de información y la carga cognitiva, están relacionados con los movimientos sacádicos y las fijaciones. Recordemos que el objetivo general de este estudio, era determinar si los valores de estos parámetros son indicadores del nivel de dificultad de una pregunta y los modelos tácitos que en esta aparecen. Además, se quiso verificar si las diferencias que se encontraron entre las preguntas y los grupos (de Matemáticas y de Ciencias), en los resultados obtenidos por criterios subjetivos y conductuales, también serían identificadas por los parámetros de seguimiento ocular.

\section{FIJACIONES}

El promedio de duración de la fijación fue significativamente, diferente para algunas preguntas [F $(7.364)=65.043, p<0,001]$. El valor más alto de este parámetro, se puede observar en la pregunta n. $^{\circ} 7$ (media $=294,13$ milisegundos) que se desvía del resto de las preguntas. Este resultado se muestra por la prueba de Scheffé, que es estadísticamente significativa $(p<0,001)$ en este caso. Sin duda, en las respuestas a esta pregunta, se mostró el mayor nivel de procesamiento de los datos oculares registrados (ver Figura 5).

El valor medio del parámetro en cuestión, fue muy similar (casi igual) en ambos grupos $[F(1,52)=0,001$, $p=0,897]$, la media del grupo de Ciencias fue de 263 milisegundos y la media del grupo de Matemáticas fue de 266 milisegundos; por lo tanto, los dos grupos mostraron un nivel comparable de procesamiento de información. 
Figura 5. Promedio de duración de la fijación: distribución de los valores medios por pregunta

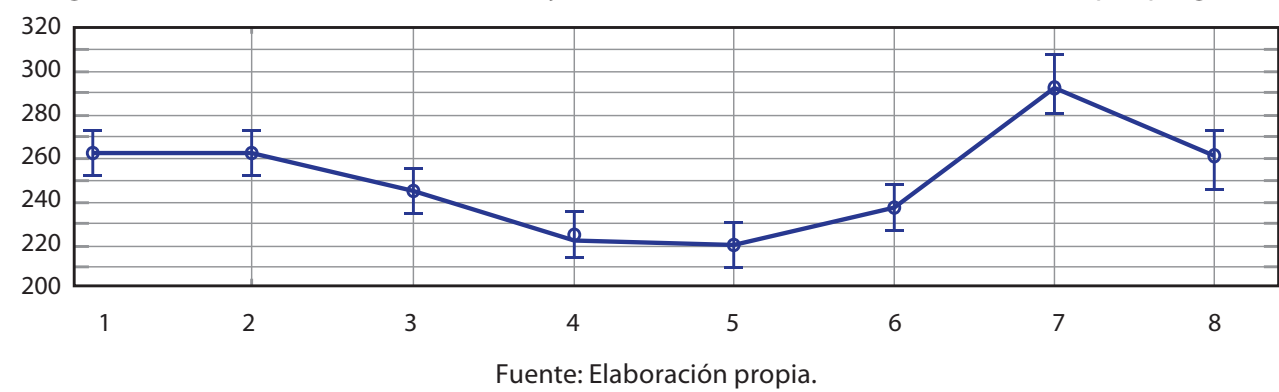

Se obtuvieron resultados similares para el parámetro de frecuencia de fijación [conteo/segundo] (ver Figura 6), indicándose una diferencia para la pregunta $n .^{\circ} 7$, para la cual este parámetro disminuyó notablemente. Esto puede interpretarse, como una prevalencia de procesos de procesamiento de información, sobre procesos de búsqueda visual. El análisis de varianza mostró diferencias significativas entre las preguntas en particular $[F(7,364)=73,91, p<0,001]$ y ausencia de diferencias, entre los grupos en cuestión $[F(1,52)=0,009, p=0,975]$.

Figura 6. Frecuencia de fijación: una distribución de los valores medios por pregunta

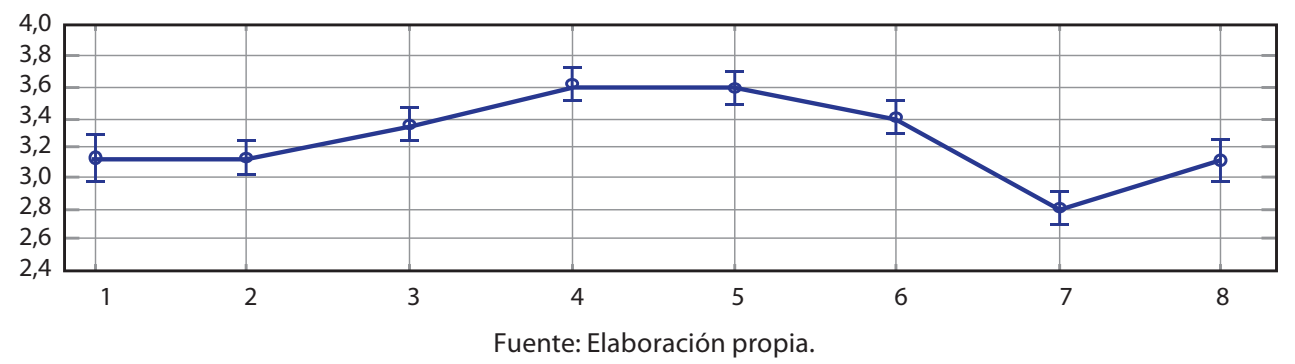

A partir del conteo total de fijaciones y los parámetros anteriores, también se pudo obtener el valor medio del tiempo total de fijación para cada pregunta. Además, se observó el parámetro de la frecuencia de movimientos sacádicos [conteo/segundo] (ver en la Figura 7 la distribución de los valores medios por pregunta). El análisis de varianza mostró diferencias significativas, entre las preguntas en particular $[\mathrm{F}(7,364)=3,32, \mathrm{p}<0,05]$.

Figura 7. Frecuencia de movimientos sacádicos: una distribución de los valores medios por pregunta

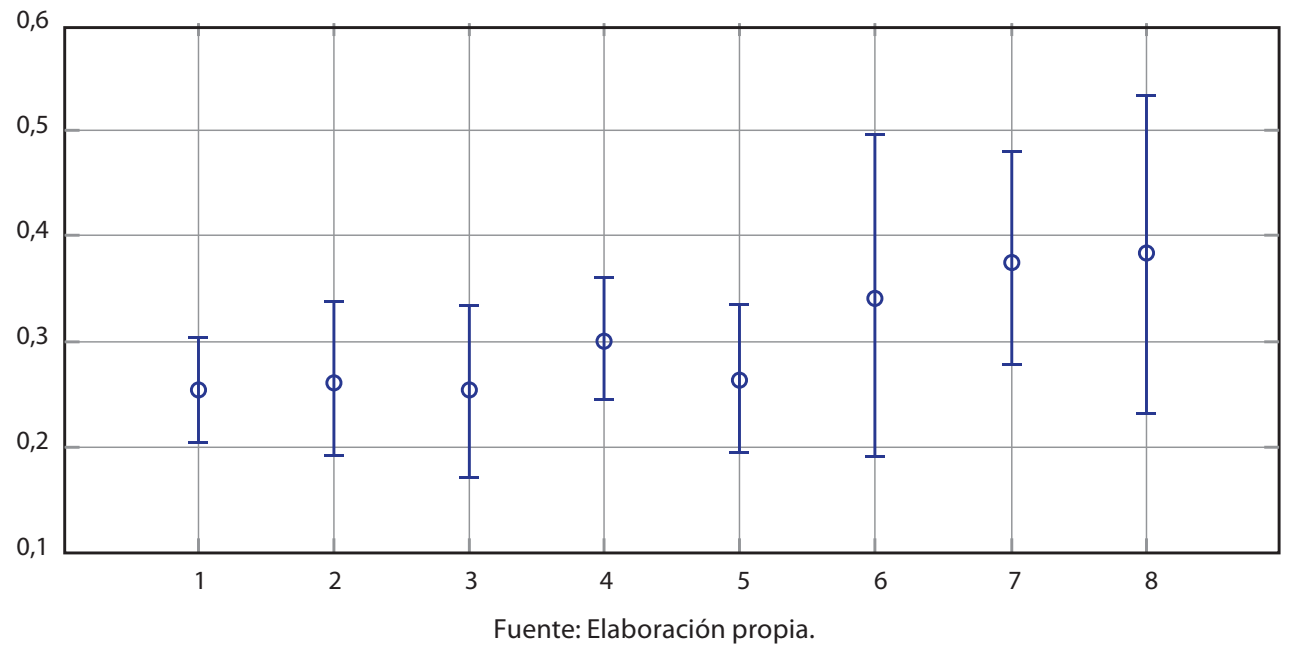


Por último, se observó el conteo total de movimientos sacádicos. En la Figura 8 se muestra la distribución de los valores medios de este parámetro por pregunta, mostrando las diferencias entre ambos grupos. El análisis de varianza mostró que no hubo diferencias significativas ni entre las preguntas en particular, ni entre los grupos de Matemáticas y de Ciencias.

Figura 8. Distribución de los valores medios del conteo de movimientos sacádicos por pregunta-diferencias entre los grupos: estudiantes de Matemáticas y estudiantes de Ciencias

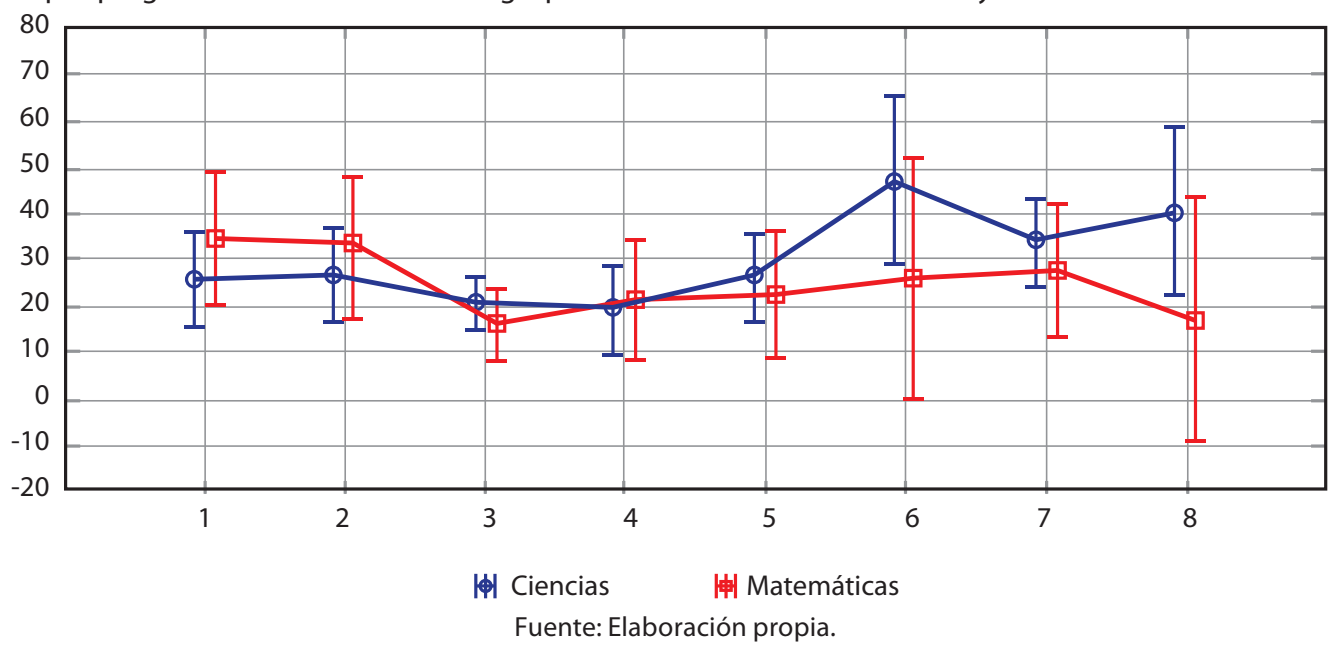

\section{CORRELACIONES}

Se determinó si existían correlaciones entre la evaluación del nivel de dificultad de la pregunta (criterio 1), el porcentaje de respuestas correctas obtenidas (criterio 2), la presencia de modelos tácitos y los parámetros discutidos anteriormente (frecuencia y conteo de movimientos sacádicos y frecuencia, duración promedio y conteo de fijaciones).

Como se presenta en la Tabla 6, las correlaciones de los parámetros de fijación y de la frecuencia de los movimientos sacádicos, con el criterio subjetivo sobre el nivel de dificultad de la pregunta (criterio 1) son bastante fuertes, aunque solo resultaron ser significativas en algunos casos. En particular, estas correlaciones muestran que, mientras mayor fue la frecuencia de movimientos sacádicos (FMS) y el promedio de duración de las fijaciones (PDF), se percibió una mayor dificultad de la pregunta.

También, se puede notar que la frecuencia de movimientos sacádicos (FMS) tuvo correlaciones fuertes significativas, con el nivel de dificultad percibido, el porcentaje de respuestas correctas obtenido y la presencia de modelos tácitos en cada pregunta. Sus correlaciones con el primer y el tercer parámetros fueron positivas, lo que indica que mientras mayor fue esta frecuencia, se percibió un mayor nivel de dificultad y hubo mayor presencia de modelos tácitos. Por otra parte, las correlaciones negativas de esta frecuencia con el porcentaje de respuestas correctas, señalan que mientras mayor fue el porcentaje de respuestas correctas, se obtuvo una menor frecuencia de movimientos sacádicos. A partir de todo lo anterior, se determinó que esta frecuencia es un indicador del nivel de participación, en el procesamiento de la información. 
TABLA 6

Coeficiente de correlación de Pearson para los parámetros de seguimiento ocular seleccionados y la evaluación del nivel de dificultad, porcentaje de respuestas correctas y modelos tácitos

\begin{tabular}{lcccccc} 
& & CMS & FMS & CF & FF & PDF \\
Dificultad subjetiva & Todos & 0,26 & $0,76^{*}$ & $-0,61$ & $-0,57$ & 0,54 \\
& GC & 0,25 & $0,74^{*}$ & $-0,60$ & $-0,58$ & 0,54 \\
& GM & $-0,14$ & $0,86^{*}$ & $-0,6^{*}$ & $-0,54$ & 0,48 \\
Porcentaje respuestas correctas & Todos & $-0,48$ & $-0,83^{*}$ & 0,59 & 0,05 & 0,00 \\
& GC & $-0,49$ & $-0,79^{*}$ & 0,55 & $-0,03$ & 0,07 \\
\hline Modelos tácitos & GM & 0,29 & $-0,89^{*}$ & $0,67^{*}$ & 0,37 & $-0,31$ \\
\hline
\end{tabular}

Nota: CMS - Conteo de movimientos sacádicos; FMS - Frecuencia de movimientos sacádicos (conteo/segundo); CF - Conteo de fijaciones; FF - Frecuencia de fijaciones (conteo/segundo); PDF - Promedio de duración de las fijaciones (milisegundos); GC-Grupo de Ciencias, GM - Grupo de Matemáticas; ${ }^{*} \mathrm{p}<0,05$.

Fuente: Elaboración propia.

El valor negativo de los coeficientes de correlación del nivel de dificultad, según el criterio 1 (evaluación subjetiva), con el conteo y la frecuencia de fijaciones, significa que a mayor conteo y frecuencia de fijaciones, le correspondió un menor nivel de dificultad percibido para una pregunta. Esto puede interpretarse, como una disminución de la actividad oculomotora en situaciones de aumento del nivel de dificultad de la tarea cognitiva. Encontramos, simultáneamente, una correlación positiva del nivel de dificultad según el criterio 2 (porcentaje de respuestas correctas) y el conteo de fijaciones, que además, es significativa en el caso del grupo de Matemáticas. Además, la presencia de modelos tácitos, se correlaciona negativamente de manera fuerte y significativa, con el conteo de fijaciones. Esto, nuevamente, podría interpretarse como una disminución de la actividad oculomotora, en situaciones de aumento del nivel de dificultad de la tarea cognitiva. En cualquier caso, los resultados parecen indicar que estos parámetros son una medida del nivel de dificultad de una pregunta, que implica la aparición de modelos tácitos.

Por último, en la Tabla 7 mostramos otros parámetros del movimiento ocular, que se correlacionaron con ambos criterios del nivel de dificultad de una pregunta. No hubo fuertes correlaciones entre la longitud total de la ruta de exploración y los criterios del nivel de dificultad. Se observan correlaciones negativas entre la evaluación subjetiva del nivel de dificultad y los tiempos totales de fijación y de respuesta, lo que resulta contradictorio y refuerza la subjetividad de este criterio.

Además, se tuvo una correlación positiva fuerte del porcentaje de respuestas correctas, que refleja el nivel de dificultad de manera objetiva, con el tiempo de ejecución de la tarea, la longitud de la ruta de exploración y la duración total de la fijación, que en algunos casos, fue significativa. Similarmente, se observó que la presencia de modelos tácitos se correlaciona negativamente, con todos estos parámetros. Todo lo anterior, señala que el tiempo de respuesta y los parámetros de actividad ocular derivados, son indicadores del nivel de dificultad asociada a la complejidad de modelos tácitos que aparecen en cada caso y nos reafirma, la interpretación de este fenómeno como una disminución de la actividad oculomotora, en situaciones de aumento del nivel de dificultad de la tarea cognitiva. 
TABLA 7

Coeficiente de correlación de Pearson para los parámetros de seguimiento ocular seleccionados y la evaluación del nivel de dificultad, porcentaje de respuestas correctas y modelos tácitos

\begin{tabular}{lcccc} 
& & TTR & TTF & LT \\
& Todos & $-0,42$ & $-0,38$ & $-0,22$ \\
Dificultad subjetiva & GC & $-0,41$ & $-0,37$ & $-0,21$ \\
& GM & $-0,49$ & $-0,41$ & $-0,29$ \\
\hline Porcentaje respuestas correctas & Todos & $0,65^{*}$ & 0,59 & 0,25 \\
& GC & $0,65^{*}$ & 0,59 & 0,22 \\
\hline Modelos tácitos & GM & 0,59 & 0,52 & 0,37 \\
\hline
\end{tabular}

Nota: TTR - Tiempo total de resolución(milisegundos); TTF - Tiempo total de fijación (milisegundos); LT - Longitud total de la ruta de exploración visual (pixels); GC-Grupo de Ciencias, GM - Grupo de Matemáticas; * p <0,05

Fuente: Elaboración propia.

Se nota también, que el tiempo total de resolución y los parámetros de actividad ocular derivados, se correlacionaron positivamente, con la evaluación objetiva del nivel de dificultad de la pregunta, a través del porcentaje de respuestas correctas. Por último, la relación entre la longitud total de la ruta de exploración, el tiempo total de resolución y la duración total de fijaciones se presenta en la Figura 7. El análisis no mostró diferencias significativas entre los grupos.

Figura 9. Comparación de los parámetros de seguimiento ocular seleccionados por pregunta

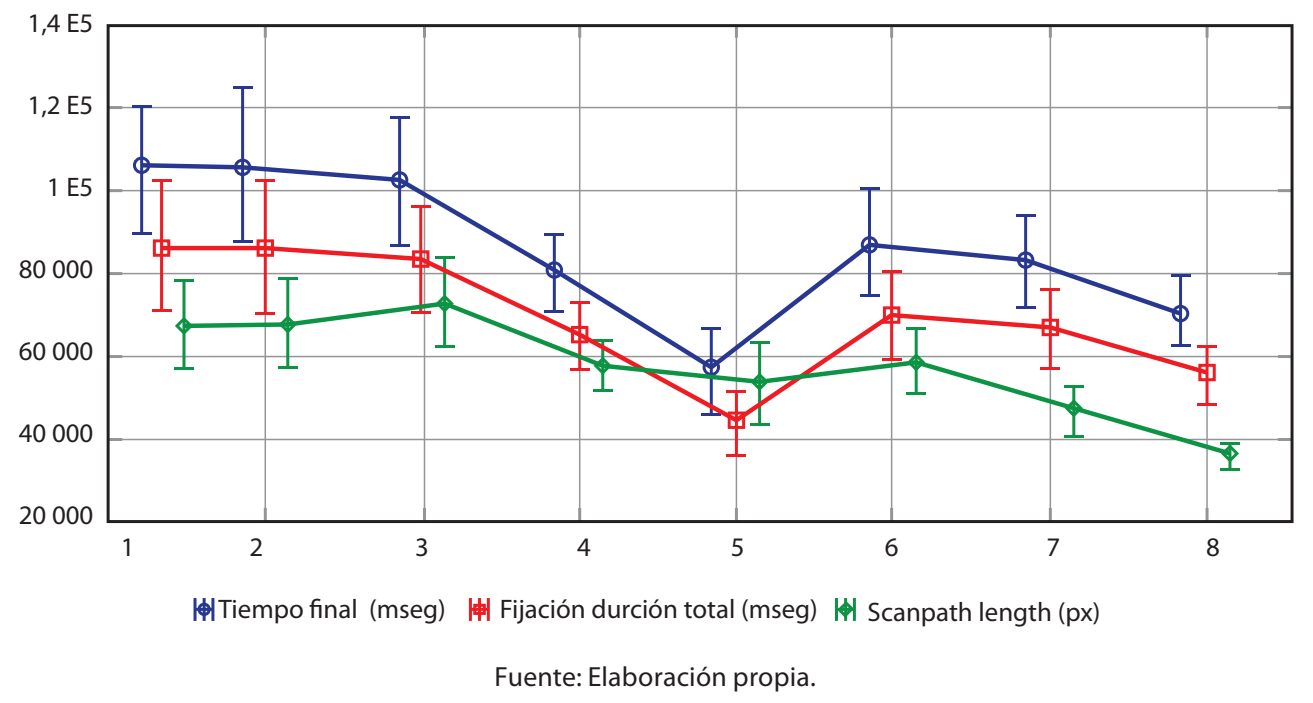




\section{SÍNTESIS Y REFLEXIONES FINALES}

Sintetizando los resultados del análisis realizado, claramente se observó una correlación fuerte y negativa, entre la mayoría de los parámetros de la actividad ocular medidos, considerados indicadores del esfuerzo mental, y la evaluación subjetiva sobre el nivel de dificultad de las preguntas. Esta evaluación está relacionada con la percepción del individuo, y está influenciada por muchos factores. Por otra parte, la presencia de modelos tácitos está relacionada con modos inconscientes de pensar de forma simplificada y es, precisamente, uno de estos factores que influye en esta evaluación subjetiva. El porcentaje objetivo (de respuestas correctas) verifica (negativa o positivamente) esta percepción subjetiva y la presencia de estos modelos, y según lo observado, es la medida más objetiva del nivel de dificultad presente.

Por otra parte, se concluye que dicho porcentaje de respuestas correctas tuvo una fuerte correlación, positiva o negativa, y en ocasiones significativa, con la mayoría de los parámetros de la actividad ocular que fueron medidos. Teniendo en cuenta todas estas correlaciones, estos parámetros podrían ser considerados indicadores del nivel de dificultad de una pregunta, en este caso. Esto, junto al hecho de que hubo una correlación positiva, fuerte y significativa, entre este porcentaje de respuestas correctas y la presencia de modelos tácitos, permite concluir, según lo planteado en el objetivo general de la investigación, que los parámetros de la actividad ocular medidos en este estudio, constituyen un índice del nivel de dificultad del modelo tácito presente en la actividad. Se puede destacar, que los resultados obtenidos fueron compatibles con los resultados de otros experimentos, en los que se han confirmado valores similares de los parámetros, en situaciones de carga cognitiva aumentada (ver por ejemplo, Debue \& Leemput, 2014) y con investigaciones realizadas en los últimos años, que afirman que las mediciones de estos parámetros son indicadores del nivel de dificultad de los problemas matemáticos (Susac et al., 2014).

El análisis mostró una presencia de diferencias significativas entre los dos grupos (grupo de Ciencias y grupo de Matemáticas), en cuanto a los dos criterios utilizados: el estudiantado del grupo de las carreras de Ciencias encontró las tareas más difíciles, y su porcentaje de respuestas correctas, fue más de dos veces menor que la del estudiantado del grupo de Matemáticas.

Se argumenta que la incorporación de este tipo de técnicas neurocientíficas a nuestro quehacer investigativo, en el campo de las ciencias de la educación, es especialmente útil y provechosa, sobre todo en el estudio de modelos como estos, que aparecen, comúnmente, en la resolución de tareas que requieren un procesamiento de información a un nivel superior e involucran, diferentes mecanismos cognitivos, que al ser inconscientes, son tan difíciles de estudiar de manera directa. La novedad de estas técnicas viene dada también, por su aporte metodológico, y los nuevos insumos que brinda para el desarrollo de nuevas investigaciones a partir de los hallazgos realizados.

Como reflexión final, se considera que los resultados de este tipo de investigación podrían tener relevancia en la transformación de nuestra práctica docente. El estudio de modelos tácitos relacionados con numerosos conceptos, y sus niveles de dificultad, deberían ser temas relevantes a la hora de construir criterios de escala y calificación, así como propuestas didácticas para las salas de clases, encaminadas a que el estudiantado se haga consciente de la presencia de estos modelos, contribuyendo a mejorar, de esta manera, los procesos de aprendizaje.

\section{Agradecimientos}

Las autoras agradecen el soporte técnico brindado por la compañía Tobii Pro Latinoámerica para la realización de este trabajo, desarrollado como parte de la Tesis Doctoral en Educación Matemática de Tamara Díaz-Chang, y parcialmente financiado por la Dirección de Investigación de la Vicerrectoría de Investigación y Postgrado, Universidad de Los Lagos, D.U. 430. 
ARRIGO, G. Y D'AMORE, B. (2004). Otros hallazgos sobre los obstáculos en la comprensión de algunos teoremas de Georg Cantor. Educación Matemática, 16, 2, 5-20.

Bachelard, G. (2004). La formación del espíritu científico. México: Siglo XXI. (Publicado en 1938).

BACKS, R. y WALRATH, L. C. (1992). Eye movement and pupillary response indices of mental workload during visual search of symbolic displays. Applied Ergonomics, 23 (4), 243-254.

BALAJ, B. y SZUBIELSKA, M. (2004). The influence of catalog description, listening on visual scanning of paintings. En S. Grucza, M. Pluzyczka, P. Soluch (Eds.), Widziane inaczej.Z. polskich badan eyetrackingowych, 77-90.

BeLmonte, J. L. y SierRA, M. (2011). Modelos intuitivos del infinito y patrones de evolución nivelar. Revista Latinoamericana de Investigación en Matemática Educativa, 14(2), 139-171.

Bolzano, B. (1991). Las paradojas del infinito. D.F., México: Universidad Nacional Autónoma de México.

D’Amore, B. (2011). La didáctica del infinito matemático. En AA. VV., Memorias del XXIV Coloquio Distrital de Matemáticas y Estadística, Bogotá, Colombia. CD. ISBN 978-958-57050-0-5, 21-29.

D'AMORE, B. y MARTINI, B. (1997). Contrato didáctico, modelos mentales y modelos intuitivos en la resolución de problemas escolares típicos. Números, 32, 26-32.

Debue, N. y Leemput, C. (2014). What does german load mean? An empirical contribution to the cognitive load theory. Frontier in Psychology, 5, 1099.

Día-Chang, T. y ARredondo, E. H. (2021a). Aquiles, la tortuga y los modelos tácitos. (En revisión) Enseñanza de las Ciencias.

Diaz-Chang, T. y ArRedondo, E. H. (2021b). Análisis de cuestionarios en el estudio de modelos tácitos relacionados con el infinito matemático. (En revisión) Revista Conrado.

Dubinsky, E.; WelLeR, K.; MC Donald, M. y BRown, A. (2005). Some historical issues and paradoxes regarding the concept of infinity: An APOS-based analysis. Educational studies in Mathematics, 58, 335-359.

FisCHBeIN, E. (2001). Tacit models and infinity. Educational studies in Mathematics, 48, 309-329.

Francuz, P. (2013). Imagia. W kierunku neurokognitywnej teorii Obrazu. Lublin: KUL.

GoldBerG, H. J.; Kotval, X. P. (1999). Computer interface evaluation using eye movements: methods and constructs. International Journal of Industrial Ergonomics, 24, 631-645.

Henderson, J. M., Holuingworth, A. (1998). Eye Movements During Scene Viewing: An Overview. En G. Underwood (Ed.) Eye Guidance in Reading and Scene Perception, 269-294.

MARSHALL, S. P. (2002). The Index of Cognitive Activity: Measuring Cognitive Workload. Proceedings of the $7^{\text {th }}$ IEEE Conference on Human Factors and Power Plants.

May, J. G., Kennedy, R. S., Williams, M. C., Dunlop, W. P., Brannan, J. R. (1990). Eye movement indices of mental workload. Acta Psychologica, 75, 75-89.

Mena-Lorca, A.; Mena-Lorca, J.; Montoya-Delgadillo, E.; Morales, A. y Parraguez, M. (2015). El obstáculo epistemológico del infinito actual: Persistencia, resistencia y categorías de análisis. RELIME, 18 (3), 329-358.

Poole, A. y BALL, L. J. (2006). Eye Tracking in $\mathrm{HCl}$ and Usability Research. Encyclopedia of Human-Computer interaction, 211-219.

RAMANAUSKAS, N. (2006). Calibration of video-oculographical eye-tracking system. Electronics and Electric Engineering, 8 (72), 65-68. 
RAYNER, K. (1998). Eye movement in reading and information processing: 20 years of research. Psychological Bulletins, 124(3), 372-422.

Soluch, P. y TARnOwskl, A. (2013). O metodologii badan eyetrackingowych. Lingwistyka Stosowana, 7, 115-134.

Steinman, R. M. (2004) Gaze control under natural conditions. En L. M. Chalupa y J. S. Werner (Eds.) The Visual Neurosciences, Cambridge: MIT Press, 1339-1356.

Strohmaier, A. R.; MacKay, K. J.; Obersteiner, A. y Reiss, K. M. (2020). Eye-tracking methodology in mathematics education research: A systematic literature review. Educational Studies in Mathematics, 104, 147-200.

Susac, A.; Bubic, A.; Kaponja, J.; Planinic, M. y Palmovic, M. (2014). Eye movements reveal students' strategies in simple equation solving. International Journal of Science and Mathematics Education, 12 (3), 555-577.

SWELLER, J. (1994). Cognitive load theory, learning difficulty and instructional design. Learning and Instruction, 4, 293-312.

TALL, D. (1981). Intuitions of infinity. Mathematics in School, 10, 3, 30-33.

Young, M. S. y Stanton, N. A. (2001). Mental workload: theory, measurement and application. En W. Karwowski (Eds) International encyclopedia of ergonomics and human factors. London: Taylor \& Francis, 1, 507-509. 